\title{
Surgery and Statistics: Strange Bedfellows
}

\author{
Amitav Banerjee $^{1}$ (D)
}

Received: 27 July 2021 / Accepted: 21 August 2021 / Published online: 28 August 2021

(c) Association of Surgeons of India 2021

"Surgeons know nothing but do everything..." This is a phrase from Robin Cook's medical thriller, the Godplayer. This backhand compliment captures the common perception about surgeons. Fiction tends to exaggerate the traits of real-life characters. In the history of medicine, the most temperamental have been the surgeons. It is said that the term "operation theatre" has its origins after the "theatrics" of great surgeons flaunting themselves as showmen [1].

Statistics on the other hand is abstract and unglamorous. After the action and excitement of the operation theatre, surgeons may find it dull and dry. Hectic workdays are also a deterrent to acquaint oneself with the basics of statistics.

However, to practice evidence-based medicine (EBM), surgeons need a statistical sense. For surgical teachers, a familiarity of statistical concepts will enable them to guide their postgraduate students better. Surgeons also require statistical insights to disseminate their work to the larger scientific community. The skills of Indian surgeons, often working with limited resources, are worthy of praise and so is their reputation. India is one of the favoured destinations for medical tourism from rich countries with far more resources [2].

In spite of world class care offered by our surgeons, their impact and contribution to the global surgical literature is modest [3]. The limiting factor is not creativity as our surgeons often improvise to overcome the constraints they work under but improper analysis and interpretation of data. Having started my career as an anaesthesiologist before moving on to epidemiology, I have often witnessed their ingenuity under difficult situations. I have also sensed their phobia of statistics while conducting research methodology workshops.

How do they cope with this phobia? They usually delegate the statistical portion of their research work to a

Amitav Banerjee

amitavb@gmail.com

1 Department of Community Medicine, Hospital and Research Centre, Dr. D. Y. Patil Medical College, Dr DY Patil

Vidyapeeth, Pune 411018, India statistician. There is nothing wrong in this. In fact, researchers and statisticians should collaborate from the inception of the research question. However, this is not the usual case. Statistical consultation is usually sought cursorily towards the end of the research project. At this belated stage, there may be little time for the statistician to get a proper insight into the research question essential for proper statistical handling of the data. Often both the researcher and the statistician have taken their eyes off the ball, i.e. the research question. The end result of such an association can be a series of meaningless calculations [4].

For a fruitful collaboration with the statistician, it is important to have a working knowledge of key statistical concepts and clear understanding of the research question. This interaction should commence from the planning stage.

To acquire this statistical sense, surgeons need not browse advanced textbooks on biostatistics. This is onerous and akin to learning the mechanical and electrical engineering aspects of a car in order to drive it. Just as driving a car is a matter of judgement and road sense in addition to knowledge of which gears to use during different stages of the drive, statistical concepts too is a matter of judgement along with which statistical tests to use depending on the scales of measurements and type of data in the context of the research question. The following paragraphs endeavour to familiarize the surgeon with the statistical gears required to undertake the research journey. This insight will also facilitate communication with the statistician or optimum use of statistical software.

The researcher should keep in mind that all measurements are done on a sample. An unrepresentative sample will lead to selection bias and loss of external validity, i.e. the study results cannot be extrapolated to the target population.

If the research is a case series with no control group, descriptive summary statistics may suffice without need for statistical tests of significance. These summary statistics depend on the type of data, i.e. categorical, ordinal or continuous.

Outcomes or variables such as deaths, gender, blood groups and so on can only be counted and clubbed into categories. These are categorical data. They are summarized 
with percentages or rates with $95 \%$ confidence intervals. In some measurements, there is a crude order or ranking, such as grades of muscle power, or the Glasgow Coma Scale to grade severity of acute traumatic brain injury and levels of consciousness, or grades of vesicoureteric reflux. These are called ordinal scales. Data measured with ordinal scale can be summarized using quartiles and Chi square for trend and sometimes median and range also.

The most robust measurements are made on continuous data. Variables such as height, weight, blood pressure and so on can be measured with the highest calibration. These types of data are called continuous, interval or quantitative data. They are summarized using mean and standard deviations. These data if normally distributed are subject to parametric analyses if not normally distributed and skewed, and then one uses median, range and non-parametric analyses.

When the research question involves hypothesis testing which involves statistical tests of significance, there are two or more groups, each exposed to different treatments or procedures. In observational studies, these groups are only observed by the investigator who has no role in allocation of these groups. For instance, in a study comparing smokers and non-smokers to see which group has longer hospital stay after a particular surgery, the groups smokers and non-smokers are selected based on smoking habits which is a personal choice and not controllable by the investigator.

Observational studies while having lesser ethical issues are lower on the hierarchy of evidence-based medicine (EBM) due to potential confounding factors giving rise to spurious associations. For instance, being a non-smoker may be associated with other healthy habits such as regular physical activity which is related to the non-smoking habit as well as faster post-operative recovery. If we do not control for this, we will overestimate the effect of smoking on post-operative recovery. In observational studies, these confounders have to be controlled for either in the planning stage by matching both the groups on these variables or in the analysis stage by stratified or multivariate analysis.

But these methods can only control for known confounders. There can be many unknown confounders. These limit the level of evidence from observational studies. The highest level of evidence is considered the randomized controlled trials (RCTs). The design and analysis are similar to cohort studies, i.e. following up an exposed group and a control group like in the example of smoking habit and post-operative recovery, the important difference being these groups are allocated by random allocation in which each participant in the trial has equal and mutually exclusive chance of being either in the exposed group or the control group. For ethical reason, we cannot design an RCT on allocating one group where the exposure or intervention is known to be harmful.

When two groups are compared for outcomes whether in observational studies or RCTs, hypothesis testing and statistical tests of significance come into play. The aim is not to eliminate uncertainty, which cannot be eliminated by empirical research, but to quantify it. Here comes the role of $P$ value. This value gives the probability of an event occurring just by chance. Consider comparing the outcomes in Group A with the new intervention and Group B which has been offered the standard management in vogue. Suppose we find a higher proportion of participants in Group A getting cured compared to Group B. In hypothesis testing using tests of significance, we start with the null hypothesis, i.e. we assume there is no difference in outcome between the two groups. However, we have found Group A has fared better than Group B. To eliminate the role of chance, we ascertain the probability of this occurring by chance given that the null hypothesis is true.

The type of test depends on the type of data. For outcomes measured on categorical scale, we test the difference in proportions by the Chi square test. For outcome measured in ordinal scale, Chi square for trend is used. Outcomes measured on continuous scale lend themselves to the most robust parametric tests of significance, i.e. $t$ test (for two groups) and analysis of variance or ANOVA (for more than two groups).

The interpretation of the $P$ value is similar whatever test is applied. The conventional significance level, $P<0.05$, conveys that though there may be an observed difference in the two groups, the probability of this occurring by chance is less than 5\%. For decision of whether to accept or reject the null hypothesis, this level of significance is the usual cut-off. This is also the type 1 error or alpha which means that we are setting $5 \%$ chance of wrongly concluding a difference in the two groups. Conversely, we may also fail to detect a difference in the groups when truly the groups differ. This is called the type 2 error or beta. Conventionally, it is set at 0.20 .

One must remember these are not hard and fast rules. Different levels of type 1 and type 2 errors may be set depending on the research question. For instance, if we do not have a treatment for some disease, we would not like to miss even a little improvement offered by a new drug. In such cases, we can go for a smaller type 2 error. Similarly, we should keep a low type 2 error for non-inferiority trials or equivalence studies in which a new drug under trial claims to be similar to the conventional drug.

An important point to remember is that statistics should not be considered in isolation. The research question, the methodology and statistical procedures should merge seamlessly from the planning stages of a research project. For a similar overview on research methodology, one may refer to an editorial written for anaesthesiologists [5], clinicians who share the workspace with surgeons. It is hoped that reading these two overviews will familiarize 
the surgeon interested in research to have more meaningful consultations with the statistician.

\section{Declarations}

Conflict of Interest The author declares no competing interests.

\section{References}

1. Mukherjee S (2011) The emperor of all maladies - a biography of cancer. Fourth Estate, London, p 66
2. Sharma A. Why India is becoming the finest medical tourism destination? Healthcare. April 16, 2019. Available at: https://www. entrepreneur.com/article/332336 (accessed 24 July 2021)

3. Kaushik R (2006) Indian surgical literature: The 'top 100' papers. Indian J Surgery 68:11-16

4. Wulff HR, Pedersen SA, Rosenberg R (1986) Probability and belief. In: Philosophy of medicine. Oxford: Blackwell Scientific Publications; p. 98-9

5. Banerjee A (2021) Research and the anaesthesiologist: cutting the clutter and overcoming the odds. Indian J Anaesth 65:183-185

Publisher's Note Springer Nature remains neutral with regard to jurisdictional claims in published maps and institutional affiliations. 